\title{
Aplicación de la autopsia psicológica como técnica pericial
}

\author{
Psychological autopsy aplication as an expert technique
}

Fernanda Piedad Clavijo Coronel $^{1} \&$ Marcia Catalina León Domínguez. $^{2}$

Recibido: 10-02-2019 / Revisado: 15-02-209 /Aceptado: 04-03-2019/ Publicado: 14-06-2019

\begin{abstract}
.
DOI: https://doi.org/10.33262/cienciadigital.v3i2.1.356

Inside of an investigative process in the case of violent deaths, there are multiple techniques of expert investigation that try to explain their cause. In the field of forensic psychology, there is an expert technique called Psychological Autopsy, which consists in carrying out a retrospective evaluation of the biography of a person whose death is not clarified. The purpose of this research is to determine the prevalence of the application of psychological autopsy in the investigation of doubtful deaths in the canton of Azogues. To this end, the bibliographic review was carried out, two surveys were developed, validated and applied to psychologists experts and public prosecutors respectively. The data concluded that the psychologists experts of the canton have not applied the technique, whereas only a public prosecutor has requested the technique's application in a single occasion.
\end{abstract}

Keywords: Psychological autopsy, expert technique, expert psychologists, prosecutor agent, prevalence.

1 Hospital Básico Santa Marianita de Jesús, Ecuador. Correo-e: fenny_16@ hotmail.com

2 Hospital Básico Santa Marianita de Jesús, Ecuador. Correo-e: catalinaleondominguez@gmail.com 


\section{Resumen.}

Dentro de un proceso investigativo en el caso de muertes violentas, existen múltiples técnicas de investigación pericial que tratan de explicar su causa. En el ámbito de la psicología forense, existe una técnica pericial llamada Autopsia Psicológica, la misma que consiste en realizar una evaluación de tipo retrospectivo acerca de la biografía de una persona cuya muerte no se encuentra esclarecida. El propósito de esta investigación es determinar la prevalencia de la aplicación de la autopsia psicológica en la investigación de muertes dudosas en el cantón Azogues. Para ello se realizó la revisión bibliográfica, se elaboraron, validaron y aplicaron 2 instrumentos para la recolección de información para los peritos psicólogos y agentes fiscales respectivamente. Los datos concluyeron que los peritos psicólogos del cantón no han aplicado la técnica, mientras que sólo un agente fiscal ha solicitado la aplicación de la técnica en una sola ocasión.

Palabras claves: Autopsia psicológica, técnica pericial, peritos psicólogos, agentes fiscales, prevalencia.

\section{Introducción.}

El sistema de justicia tiene la difícil tarea de investigar los casos de muerte violenta, para determinar su causa. Bajo la premisa de que los homicidas no quieren ser atrapados, tratan de ocultar evidencia e incluso intentar que el crimen parezca un suicidio. Así, surge la necesidad de complementar la investigación forense con técnicas periciales específicas para esclarecer muertes violentas.

Por otra parte, según la Organización Mundial de la Salud (OMS), más de 800.000 personas se suicidan cada año, lo que significa que la mortalidad por el suicidio es superior a la causada por la guerra e incluso el homicidio. Sin embargo, en el Ecuador, según la estadística del Instituto Nacional de Estadísticas y Censos (INEC), en el 2014 la mortalidad por agresiones (homicidio) fue superior a la producida por lesiones autoinflingidas intencionalmente o suicidio; lo más alarmante es que la tasa de mortalidad mayor a las mencionadas fue por causas mal definidas, lo que implica una falencia en el sistema de salud y de justicia.

Como primer paso para la administración eficaz de la justicia, se considera indispensable determinar si se aplica o no la técnica de autopsia psicológica en el medio en que nos desenvolveremos como peritos, es decir, en el cantón Azogues.

Los delitos contra la vida son especialmente atroces, por lo tanto, resulta coherente que sean sancionados con mayor rigurosidad. El Derecho Penal asume la responsabilidad de identificar y sancionar a quienes cometen este tipo de delitos, por lo que Zaffaroni sostiene 
que la función del derecho penal es actuar como un "sistema inteligente de filtros para contener racionalmente las pulsiones del poder punitivo" (Encalada Hidalgo, 2014).

En un sistema que tiene como premisa la inocencia del imputado, es indispensable al momento de administrar justicia, trabajar con un equipo profesional interdisciplinar, que aporte información objetiva y científica necesaria para esclarecer el hecho delictivo. He aquí la importancia de que el perito psicólogo esté capacitado para realizar la autopsia psicológica, y así cumplir el rol de auxiliar de la justicia en los casos de muerte violenta.

La autopsia psicológica como técnica pericial es utilizada para diferenciar un suicidio de un homicidio en diversos países, por lo que este estudio tiene el propósito de determinar la prevalencia de la aplicación de la técnica en el cantón Azogues.

La vida es el derecho fundamental que tiene el ser humano, puesto que es requisito indispensable para que los otros derechos puedan hacerse exigibles. Este derecho se encuentra amparado en la Constitución de la República, y es responsabilidad del Estado tutelar el bien jurídico de la vida, para proteger la existencia de sus habitantes. Es por esto que, cuando se viole este derecho, se deberá contar con los medios de prueba necesarios para encontrar la verdad de los hechos y así establecer la existencia y la responsabilidad en casos de asesinato u homicidio.

Cuando se produce una muerte en condiciones ambiguas, traumáticas, desconocidas o dudosas, cabe plantear la posibilidad de que haya sido un suicidio. Sin embargo, es indispensable investigar qué ha podido llevar a la muerte y determinar si efectivamente se trata de un suicidio o de un homicidio, asesinato o muerte accidental. De esta manera es indefectible en este trabajo, partir con la definición de las maneras de muerte.

Muerte natural: se entiende como aquella que es el resultado último de un proceso de enfermedad en el que no existe la participación de agentes externos. Su etiología es endógena o cuando es exógena debe ser espontánea, como en el caso de las infecciones (Sánchez, s.f.)

Muerte violenta: se desarrolla a través de un agente exógeno al individuo, en forma de suicidio, homicidio o accidental.

Suicidio: es el acto de ocasionarse la muerte de forma voluntaria.

Homicidio: es el acto de acabar con la vida de otra persona, ya sea de manera intencional o imprudencial.

Accidental: es un suceso eventual o acción que involuntariamente resulta en la muerte de una persona.

Asesinato: es una especie de homicidio agravado, en el cual una persona actúa con premeditación, alevosía y maldad. 
Muerte dudosa: se entiende a aquella en la que se desconoce la causa exacta de la muerte, y además se encuentra relacionada con la intervención de circunstancias externas al occiso (Gómez. \& Saenz, 2000)

Investigar una muerte violenta es un proceso complejo, por lo que el trabajo interdisciplinar enriquece la indagación y aproxima al esclarecimiento de los hechos para encontrar la verdad.

El proceso de investigación inicia con el reconocimiento del lugar de los hechos, que debe ser realizado por un equipo de peritos acreditados por el Consejo de la Judicatura.

Por su parte, el Código Orgánico Integral Penal (COIP), determina que las y los peritos deberán "ser profesionales expertos en el área, especialistas titulados o con conocimientos, experiencia o experticia en la materia y especialidad, acreditados por el Consejo de la Judicatura".

El artículo 598 del COIP, establece que "En la instrucción, cualquiera de los sujetos procesales podrá solicitar a la o al fiscal que disponga la práctica de las pericias que sean necesarias para obtener los elementos de convicción”.

Los medios de prueba, según lo establecido en el COIP, son el documento, el testimonio la pericia. El objetivo primordial de la prueba es conducir al juzgador al pleno convencimiento de los hechos y circunstancias de la infracción, así como la responsabilidad del procesado; es por eso que el COIP establece que la prueba debe tener un nexo causal entre la infracción y el sujeto procesado.

La autopsia psicológica debe considerarse una técnica pericial porque permite la investigación de los hechos a través del levantamiento de indicios, huellas psicológicas y evidencia que se constituyen en prueba con el informe pericial y su respectiva sustentación oral en la audiencia de juicio.

Autopsia psicológica. - La ausencia del testimonio de la víctima, sea por desaparición o muerte, dificulta la investigación; sin embargo, existen fuentes alternativas de información que permiten obtener datos claves para el esclarecimiento de un delito. Una técnica reconocida internacionalmente dentro de las ciencias forenses, para obtener tales datos es la autopsia psicológica.

Teresita García Pérez (2009) define a la autopsia psicológica como "el método de investigar retrospectivamente las características de la personalidad y las condiciones que en vida tuvo un individuo, teniendo como objetivo acercarse a la comprensión de las circunstancias de su muerte". Además, García Pérez (2014), conceptualiza a la autopsia psicológica como "un 
procedimiento para evaluar cómo era la víctima en vida", a través de la reconstrucción biográfica de toda la historia de vida de la persona fallecida, haciendo énfasis en: "el estilo de vida, la personalidad, estrés reciente, enfermedad mental y comunicación de ideas orientadas a la muerte, enfocándose en los últimos días y horas” del occiso.

Principios metodológicos: Para Ebert (1991, citado por Evangelina Ghe, 2015), existen algunos objetivos que persigue la autopsia psicológica, pero destaca cuatro como los más importantes.

1. Primer Objetivo: establecer la manera de muerte en casos que necesitan ser distinguidos. Las maneras de muerte son: a) natural, b) accidental, c) suicidio y, d) homicidio.

2. Segundo Objetivo: averiguar el momento y el tiempo en el cual se produjo la muerte; para ello, el investigador debe examinar acerca de diferentes facetas y situaciones de la vida del occiso y vincularlas con el hecho que desencadenó en la muerte.

3. Tercer Objetivo: en el caso de existir antecedentes referentes a intentos de suicidio, se debe obtener la información pertinente al hecho, con el propósito de poder prevenir nuevos intentos y la letalidad de los mismos llegasen a tener.

4. Cuarto Objetivo: como mecanismo terapéutico para la familia y los amigos del occiso, quienes participan de la investigación. Cumple con un rol terapéutico puesto permite expresar pensamientos y sentimientos sobre la persona fallecida y la percepción que cada uno de ellos tenía sobre la misma, generando así un efecto catártico.

Siguiendo los principios metodológicos expuestos, la técnica se desarrolla de la siguiente manera:

Análisis de la escena del crimen: Ya sea abierta o cerrada, se observa cuidadosamente la posición del cadáver, la presencia de objetos de valor y se valora si hay elementos comunes con otras víctimas. En el caso de escenas cerradas se examina la literatura, música, el mobiliario, la calidad de los objetos y el gusto con el que están dispuestos.

Las personas que conocían directamente a la víctima: Se entrevista a familiares y amigos cercanos a la víctima para inferir algún tipo de alteración psicológica.

Documentos oficiales y personales: En caso de encontrar una posible carta suicida, ésta debe ser analizada para determinar si los rasgos grafológicos pertenecen a la víctima y si hubo o no presión para que la escriba. Además, se revisan documentos personales de la víctima como diarios, cartas, correspondencia, expedientes laborales o escolares, así como reportes médico-legales, exámenes toxicológicos y obras científicas o literarias de su autoría.

Modelo de Autopsia Psicológica Integrado - MAPI

El Modelo de Autopsia Psicológica Integrado (MAPI) fue desarrollado en 1990, por la Dra. Teresita García Pérez, especialista en psiquiatría del Instituto de Medicina Legal de la Ciudad 
de la Habana. Es un método estructurado y sistematizado para llevar a cabo una Autopsia Psicológica, el cual permite realizar un diagnóstico pericial acertado tanto en víctimas de suicidio, homicidio, así como de accidentes (Burgos, 2006)

El MAPI es un instructivo basado en preguntas de respuesta cerrada, con el objetivo de evitar la inclusión de elementos subjetivos, por parte del investigador, en la valoración de cada caso y, además, para hacerlo verificable por terceras personas, (Rodríguez, 2001)

La Autopsia Psicológica como técnica pericial puede vincularse al Derecho penal, civil y laboral, pues permite determinar: el estado mental de víctimas de muertes violentas, la capacidad testamentaria e incluso en casos de accidentabilidad laboral. En el campo de la criminología la autopsia psicológica se constituye como el método que permite el estudio del delito, el delincuente y la víctima (Blasco, J., Blasco, C., Ventura, M. \& Canet, A., 2004)

\section{Metodologia.}

El presente estudio es de carácter exploratorio debido al objeto de estudio y de alcance descriptivo en relación a los objetivos planteados.

La investigación es de tipo cuantitativo debido al objeto de estudio y la metodología necesaria.

El universo a estudiar son los peritos psicólogos y agentes fiscales a nivel nacional; siendo la muestra del presente estudio los del cantón Azogues.

Criterios de inclusión y exclusión:

\section{Inclusión}

- Psicólogos Peritos del cantón Azogues.

- Agentes fiscales del cantón Azogues.

\section{Exclusión}

- Peritos psicólogos que no sean del cantón Azogues.

- Psiquiatras y demás profesionales de la salud mental.

- Agentes fiscales que no pertenezcan al cantón Azogues.

- Técnicas de recolección y análisis de la información

- Revisión bibliográfica.

- Elaboración, validación y aplicación de una encuesta.

- Elaboración de una base de datos en Microsoft Excel para el procesamiento y análisis de la información.

\section{Procedimiento}

Se procedió a realizar una investigación sistematizada de bibliografía actualizada referente a la técnica y su aplicación.

Se elaboraron dos encuestas de 6 preguntas para los agentes fiscales y los peritos respectivamente. 
Para la validación del instrumento se procedió a realizar una prueba piloto con 30 personas, 15 abogados y 15 psicólogos clínicos del cantón Azogues. Los datos obtenidos fueron procesados en Microsoft Excel para determinar la confiabilidad y validez de la encuesta.

La confiabilidad, entendida como la estabilidad o consistencia de los resultados obtenidos, se midió a través del cálculo del coeficiente Alpha de Cronbach, obteniendo 0,97, lo que significa que existe confiabilidad en la medición.

Previo a la aplicación de la encuesta, se informó a los participantes el objeto de investigación y se pidió su autorización expresa para brindar información mediante la firma del consentimiento informado.

La Fiscalía del cantón Azogues cuenta con ocho agentes fiscales, y una perito psicóloga.

El Consejo de la Judicatura cuenta con dos peritos psicólogos en la unidad de familia. Además, existe un psicólogo perito privado en el cantón.

Durante dos días se realizó la aplicación de la encuesta a los participantes antes mencionados, con excepción de un agente fiscal que se encontraba indispuesto.

Una vez obtenidos los resultados, se registraron en la base de datos en Microsoft Excel y se procedió a analizarlos.

\section{Resultados.}

¿Cuál es su profesión?

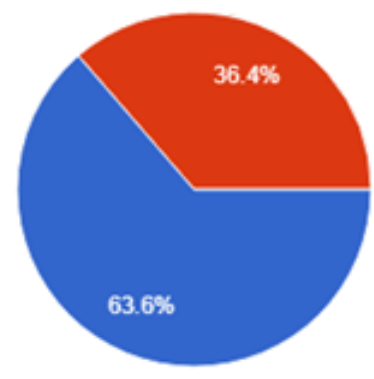

Abogado/a

Psicólogo/a

\section{Gráfico 1}

Fuente: encuestas

Elaborado por: las autoras.

Fecha: 11 de septiembre de 2018

El estudio cuenta con la participación de 7 agentes fiscales y 4 peritos psicólogos, lo que representa el $63.6 \%$ y el $36,4 \%$ respectivamente.

De los 4 peritos psicólogos, 2 pertenecen a la Unidad de Familia del Consejo de la Judicatura, por lo que no están vinculados a la investigación penal; sin embargo, se les consideró para enriquecer el estudio por la escasa cantidad de peritos del Cantón. 
¿Cuántos años ejerce su función como perito?

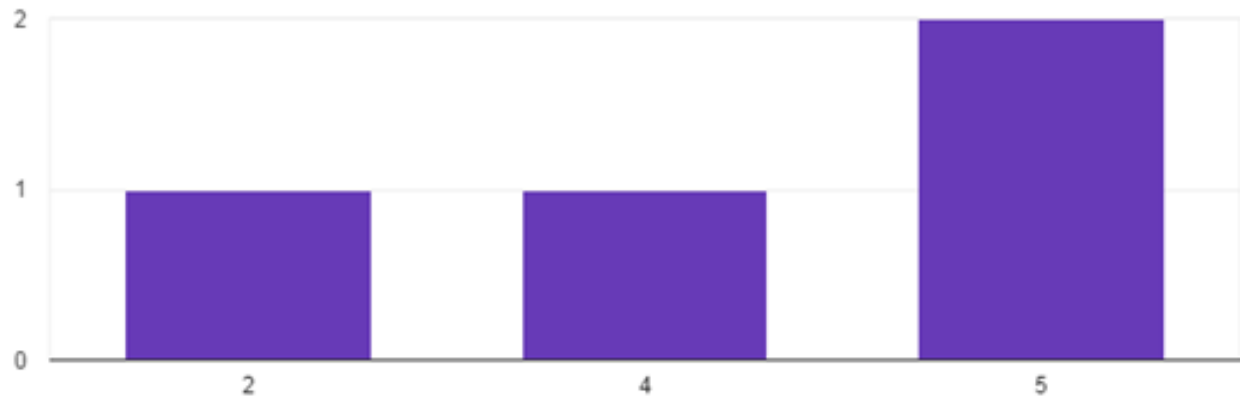

Años

\section{Gráfico 2}

Fuente: encuestas

Elaborado por: las autoras.

Fecha: 11 de septiembre de 2018

El rango de años en los que los psicólogos ejercen su función de perito oscila entre 2 a 5 años. Considerando que el tiempo mínimo requerido para la calificación como perito es de 2 años de graduados, se determina que los profesionales llevan al menos 4 años de experiencia profesional.

\section{Conozco la técnica de autopsia psicológica}

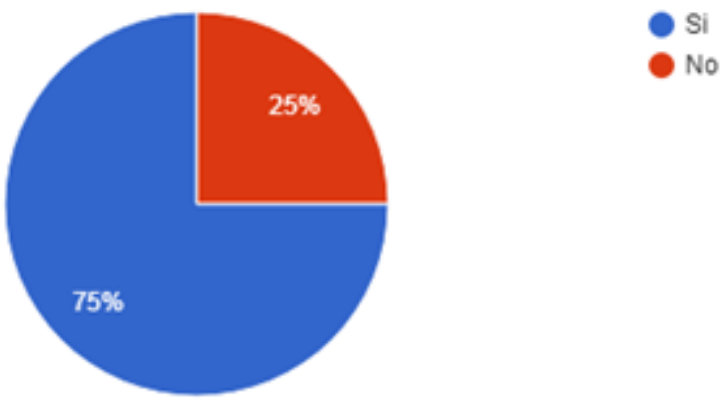

Gráfico 3

Fuente: encuestas

Elaborado por: las autoras.

Fecha: 11 de septiembre de 2018

El 75\% de los encuestados manifiesta conocer el uso de la técnica autopsia psicológica. Ya que los peritos encuestados son Psicólogos Clínicos, el 25\% desconoce la técnica al no encontrarse dentro de su malla curricular. 


\section{Distingo los casos en los que debe utilizarse la autopsia psicológica}

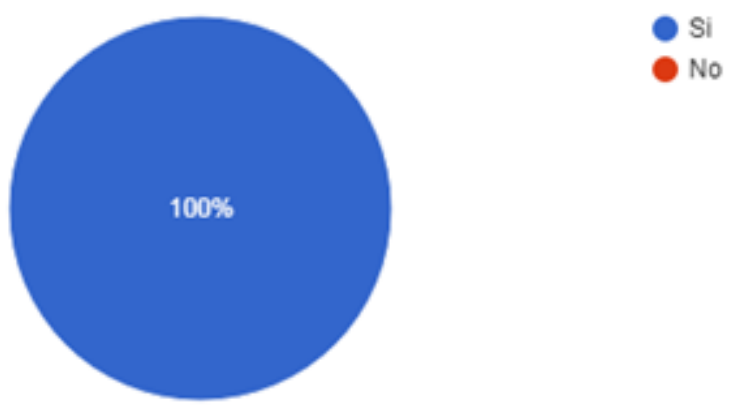

\section{Gráfico 4}

Fuente: encuestas

Elaborado por: las autoras.

Fecha: 11 de septiembre de 2018

El $100 \%$ de la población encuestada asegura distinguir los casos en los que se debe utilizar la técnica de autopsia psicológica. Lo que resulta contradictorio con los resultados de la pregunta anterior, pues afirman identificar los casos en los que debe usarse una técnica desconocida por el $25 \%$ de encuestados.

\section{Dentro de mi práctica profesional he implementado el uso de la técnica}

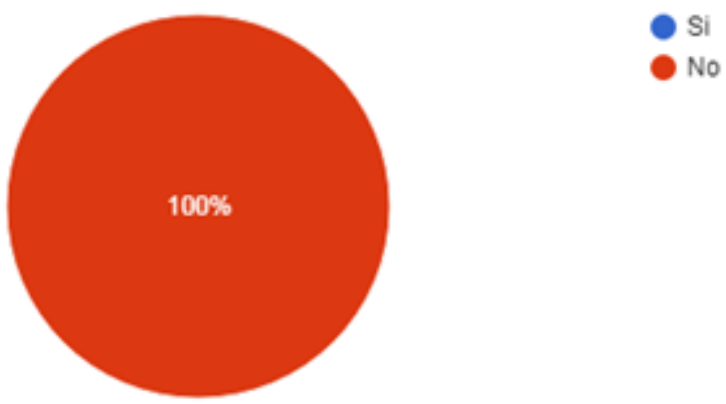

\section{Gráfico 5}

Fuente: encuestas

Elaborado por: las autoras.

Fecha: 11 de septiembre de 2018

El 100\% de peritos psicólogos que trabajan dentro de la Fiscalía y Consejo de la Judicatura del cantón Azogues, no ha empleado la técnica de autopsia psicológica. Incluso aquellos 
peritos que afirman conocer la técnica, no la han usado, pues es un requerimiento de los abogados defensores y agentes fiscales.

Considero importante el uso de la autopsia psicológica dentro de mi campo laboral.

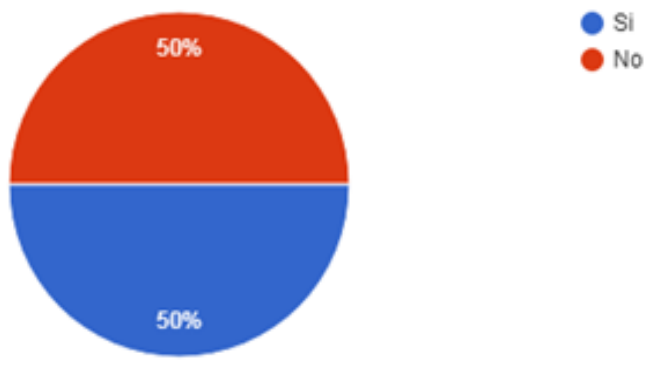

\section{Gráfico 6}

Fuente: encuestas

Elaborado por: las autoras.

Fecha: 11 de septiembre de 2018

El 50\% de peritos psicólogos encuestados consideran importante el uso de la técnica autopsia psicológica. Este resultado se interpreta como óptimo, pues el $50 \%$ de peritos encuestados pertenecen a la unidad de familia, por lo que en el desempeño de sus funciones no se encuentra la investigación de casos de muertes violentas.

\section{¿Cuántos años ejerce su función como agente fiscal?}

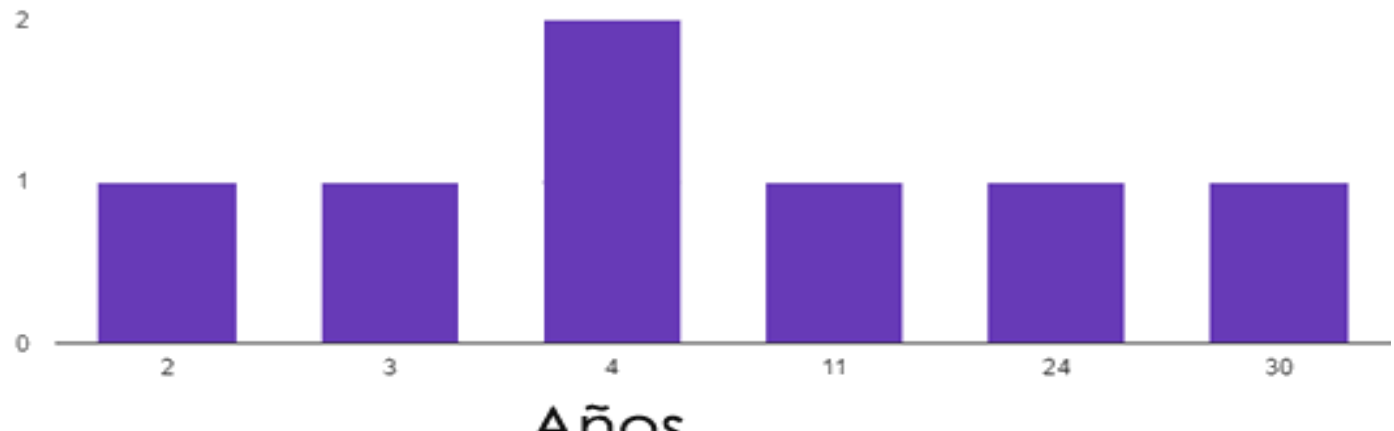

\section{Gráfico 7}

Fuente: encuestas

Elaborado por: las autoras.

Fecha: 11 de septiembre de 2018

El récord fiscal oscila entre los 2 y 30 años, lo que demuestra la heterogeneidad de la muestra. 


\section{Conozco la técnica de autopsia psicológica}

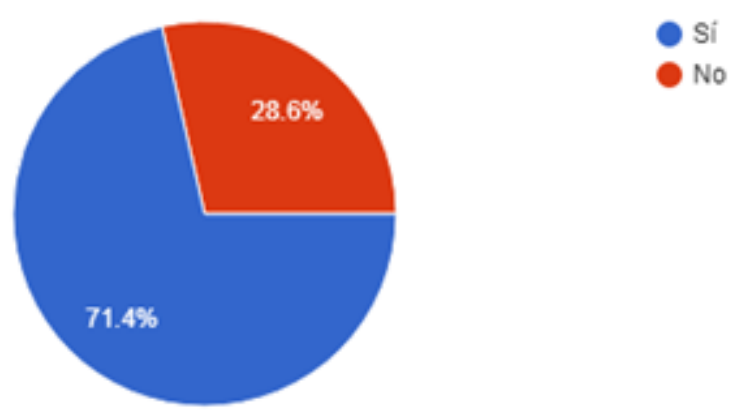

\section{Gráfico 8}

Fuente: encuestas

Elaborado por: las autoras.

Fecha: 11 de septiembre de 2018

El 71,4\% de agentes fiscales del cantón Azogues conocen la técnica de autopsia psicológica, mientras que el 28,6\% la desconoce. Cabe mencionar que durante la aplicación de la encuesta, los agentes fiscales mencionaron la importancia de tener peritos capacitados para aplicar la autopsia psicológica, y sugirieron se les capacite en la técnica para poder solicitarla.

\section{Distingo los casos en los que debe utilizarse la autopsia psicológica}

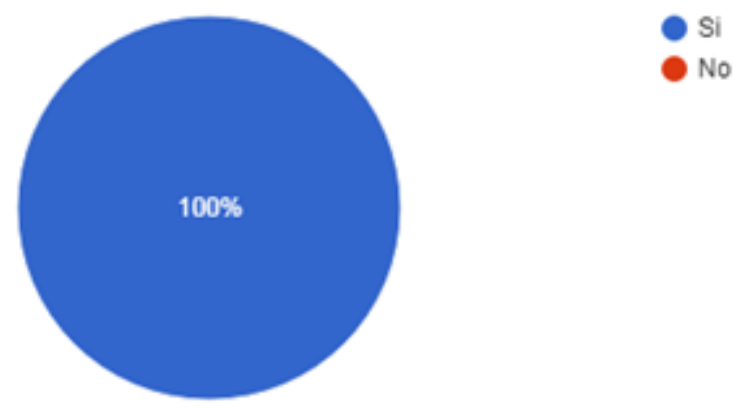

\section{Gráfico 9}

Fuente: encuestas

Elaborado por: las autoras

Fecha: 11 de septiembre de 2018

Todos los agentes fiscales encuestados distinguen los casos en los que debe utilizarse la técnica; al igual que los peritos psicólogos encuestados, a pesar de que no todos conocen la técnica, afirman distinguir los casos en los que es necesaria. 
Dentro de mi práctica profesional he solicitado el uso de la técnica

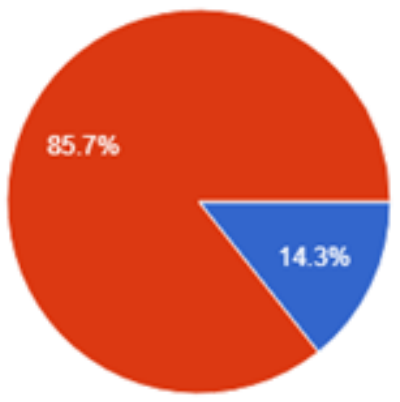

\section{Gráfico 10}

Fuente: encuestas.

Elaborado por: las autoras.

Fecha: 11 de septiembre de 2018

El 85,7\% de encuestados NO ha solicitado la aplicación de la técnica. Entre los participantes, solo un agente fiscal manifiesta haber solicitado la técnica.

Considero importante el uso de la autopsia psicológica dentro de mi campo laboral

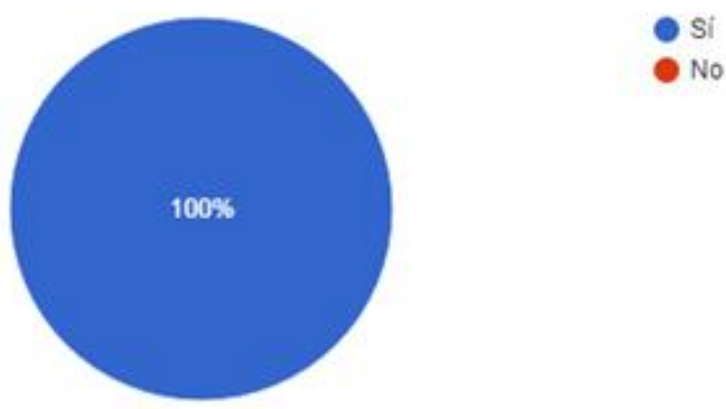

\section{Gráfico 11}

Fuente: encuestas

Elaborado por: las autoras.

Fecha: 11 de septiembre de 2018

El $100 \%$ de agentes fiscales consideran importante el uso de la técnica en su campo laboral. A pesar de desconocer la técnica o de no haberla solicitado, concuerdan en que es necesaria en su accionar. 


\section{Conclusiones.}

- La autopsia psicológica ha sido reconocida como una técnica de gran aporte dentro del proceso pericial, pues a partir de la fundamentación teórica analizada se considera la importancia de la misma en la resolución de casos vinculados a muertes violentas, específicamente en la diferenciación entre un homicidio y un suicidio. Andrade, Téllez \& Giraldo (2013) también reconocen la importancia de la técnica como una "herramienta evaluativa", sin embargo hacen énfasis en registrar a la Autopsia Psicológica no sólo como un instrumento descriptivo, sino como también como un "dispositivo de prevención", puesto que si se la analiza la técnica a partir de un enfoque longitudinal en el análisis de datos, es posible detectar factores de riesgo asociados al acto suicida, pues este tipo de muerte es el que mayor relevancia posee en la investigación en la que versa la Autopsia Psicológica.

- Dentro de la presente investigación de campo, se encontró que en relación al grado de conocimiento por parte de los agentes fiscales del cantón Azogues, el 71,4\% refieren conocerla, aunque su aplicación es prácticamente nula dentro de las investigaciones por muertes violentas, según refieren los encuestados. Datos que se corresponden con la investigación de tesis de pregrado titulada "Autopsia Psicológica y los delitos contra la vida" (Aponte, 2017), en donde la autora aplica una encuesta a Agentes Fiscales y Abogados en libre ejercicio de la ciudad de Ambato, cuyos datos arrojan que el $85 \%$ de la población investigada refiere conocer acerca de la técnica. De igual manera en dicha investigación, un pequeño porcentaje $(14,4 \%)$ de la población encuestada ha recurrido a la aplicación de la técnica dentro de su campo de trabajo.

- En relación a la aplicación de la Autopsia Psicológica por parte de los peritos psicólogos del cantón Azogues, se ha encontrado que ninguno de ellos ha aplicado la técnica. Esto puede deberse a la falta de formación académica sobre la técnica y a la par sumarse a que profesionales fiscales no requieren el uso de la misma, según refieren los datos de esta investigación. Dicha premisa tiene correspondencia con el trabajo de pregrado titulado "Aplicación de la Autopsia Psicológica en el Proceso Penal Colombiano" (Gómez \& Sáenz, 2000) en cuyo análisis las autoras reconocen que las autoridades y profesionales involucrados en las valoraciones periciales, no incluyen el uso de la técnica Autopsia Psicológica, cuanto menos se encuentra contemplado dentro de algún código legislativo en su medio, al igual que en nuestro país, en la búsqueda bibliográfica en varios códigos judicial/legislativo en el área pericial, no existe un respaldo científico-teórico sobre el tema investigado.

- Finalmente, la aplicación de la autopsia psicológica en el esclarecimiento de muertes violentas en el cantón Azogues, se reduce a una única solicitud por parte de un agente fiscal. 


\section{Referencias bibliográficas.}

Andrade, J., Cesar Humberto Téllez, C. y Giraldo, L. (2013) La Autopsia Psicológica en Colombia: Exploración Psicobiografica del Suicidio. Obtenido de Revista Internacional de Psicología ISSN 1818-1023 www.revistapsicologia.org Instituto de la Familia Guatemala Vol.12 No.02 Junio 2013. http://Users/Pc/Downloads/DialnetLaAutopsiaPsicologicaEnColombia-6161368.pdf

Aponte Sevilla, K. G. (2017). La autopsia psicológica y los delitos contra la vida. Obtenido de Universidad Técnica de Ambato: http://repositorio.uta.edu.ec/bitstream/123456789/24715/1/FJCS-DE-994.pdf

Blasco, A., Blasco, C,. Ventura,M. y Canet, A. (2004). Lla autopsia psicológica en medicina legal y forense. Obtenido de Boletín Galego de Medicina Legal e Forense $\mathrm{n}^{\circ} .13$. Noviembre 2004 http://www.agmf.es/az/La_autopsia_psicologica_en_medicina_legal_y_forense._Bl asco_JA_et_al.pdf

Burgos, A. (2006). La autopsia psicológica. Obtenido de Revista de Ciencias Jurídicas $N^{o}$ 110 (69-87) mayo-agosto 2006. https://revistas.ucr.ac.cr/index.php/juridicas/article/download/13637/12947

Código Orgánico Integral Penal. (2014). Quito.

Corea Vindas, N. (Febrero de 2017). La utilidad jurídica de la autopsia psicológica como medio de prueba en el proceso penal costarricense. Obtenido de Universidad Hispanoamericana:

http://170.246.100.106:7501/xmlui/bitstream/handle/cenit/1525/DERHE\%200378.pdf?sequence=1\&isAllowed $=\mathrm{y}$

Encalada Hidalgo, P. (5 de Febrero de 2014). Teoría constitucional del delito y el Código Orgánico Integral Penal. Obtenido de Revista Pensamiento Penal: http://www.pensamientopenal.com.ar/system/files/2014/05/doctrina38794.pdf\#view er.action=download

García Pérez, T. d. (2014). La autopsia psicológica ¿suicidio u homicidio? La Habana: Editorial Científico-Técnica.

Ghe, E. S. (2015). Autopsia psicológica: método de investigación criminal. Obtenido de Acta Académica: https://www.aacademica.org/000-015/551

Godoy, S., \& Tirira, M. (Octubre de 2018). La respuesta judicial del femicidio en Ecuador Vol. II Análisis de sentencias relativas a muertes violentas de mujeres ocurridas en 
el 2016. Obtenido de Defensoría del Pueblo Ecuador: http://repositorio.dpe.gob.ec/bitstream/39000/2206/1/VCM-DPE-018-2018.pdf

Gómez, E. y Saénz, M. (2000). Aplicación de la autopsia psicológica en el proceso penal colombiano. https://www.javeriana.edu.co/biblos/tesis/derecho/dere1/Tesis35.pdf

Instituto Nacional de Estadísticas y Censos. (2014). Principales causas de mortalidad. Obtenido de Ecuador en cifras: http://www.ecuadorencifras.gob.ec/vdatos/

Rodriguez, A. (2001). Autopsia psicológica: Una herramienta útil para el peritazgo psicológico. http://www.icdcriminal.com.ar/cont/articulos/AUTOPSIA\%20PSICOL\%C3\%93GI CA.pdf

Torre, R. (2007). La victima del crimen. Buenos Aires:DOSYUNA

Vera García, B. M., Alarcón Chavez, B. E., \& Menéndez Moreira, E. A. (2018). Intervención del psicólogo clínico frente al femicidio en la ciudad de Portoviejo. Obtenido de Dialnet: https://dialnet.unirioja.es/servlet/articulo?codigo=67199

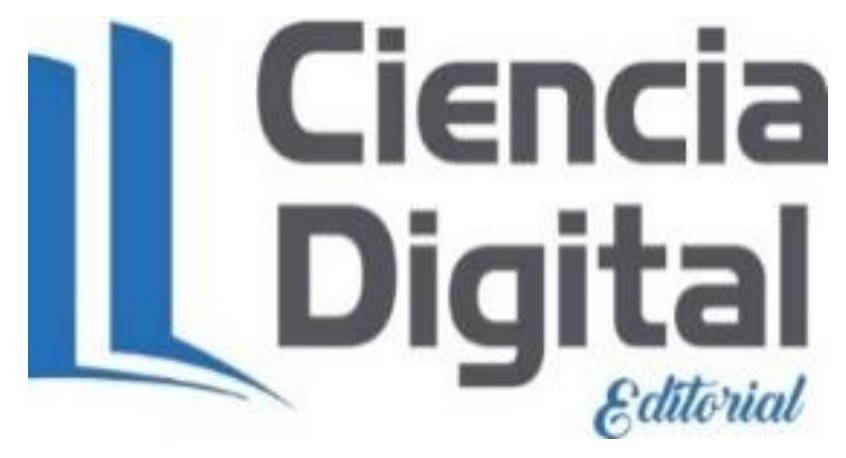


Para citar el artículo indexado.

Clavijo F \& León M. (2019) Aplicación de la autopsia psicológica como técnica pericial. Revista electrónica Ciencia Digital 3(1.1), 7-22. Recuperado desde: http://cienciadigital.org/revistacienciadigital2/index.php/CienciaDigital/article/view/356/774

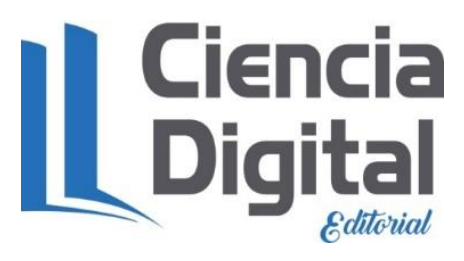

El artículo que se publica es de exclusiva responsabilidad de los autores y no necesariamente reflejan el pensamiento de la Revista Ciencia Digital.

El articulo queda en propiedad de la revista y, por tanto, su publicación parcial y/o total en otro medio tiene que ser autorizado por el director de la Revista Ciencia Digital.
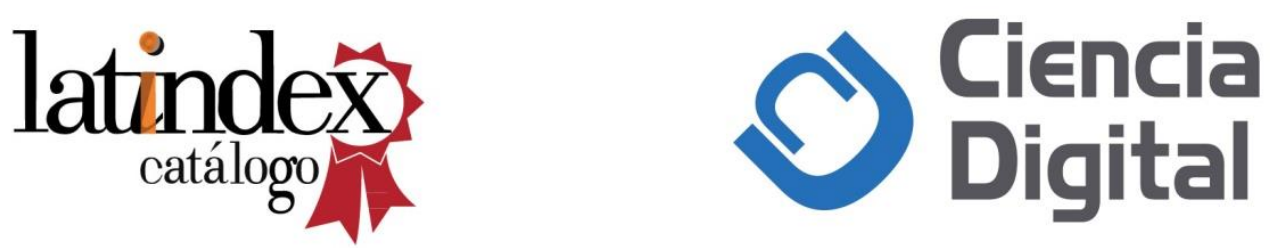